\title{
Optimality Conditions for Fuzzy Number Quadratic Programming with Fuzzy Coefficients
}

\author{
Xue-Gang Zhou, ${ }^{1,2}$ Bing-Yuan Cao, ${ }^{1}$ and Seyed Hadi Nasseri ${ }^{3}$ \\ ${ }^{1}$ School of Mathematics and Information Science, Key Laboratory of Mathematics and Interdisciplinary Sciences of Guangdong, \\ Higher Education Institutes, Guangzhou University, Guangzhou, Guangdong 510006, China \\ ${ }^{2}$ Department of Applied Mathematics, Guangdong University of Finance, Guangzhou, Guangdong 510521, China \\ ${ }^{3}$ Department of Mathematical Sciences, University of Mazandaran, P.O. Box 47415-1468, Babolsar, Iran
}

Correspondence should be addressed to Bing-Yuan Cao; caobingy@163.com

Received 16 January 2014; Accepted 27 March 2014; Published 23 April 2014

Academic Editor: Mohammad Khodabakhshi

Copyright (c) 2014 Xue-Gang Zhou et al. This is an open access article distributed under the Creative Commons Attribution License, which permits unrestricted use, distribution, and reproduction in any medium, provided the original work is properly cited.

The purpose of the present paper is to investigate optimality conditions and duality theory in fuzzy number quadratic programming (FNQP) in which the objective function is fuzzy quadratic function with fuzzy number coefficients and the constraint set is fuzzy linear functions with fuzzy number coefficients. Firstly, the equivalent quadratic programming of FNQP is presented by utilizing a linear ranking function and the dual of fuzzy number quadratic programming primal problems is introduced. Secondly, we present optimality conditions for fuzzy number quadratic programming. We then prove several duality results for fuzzy number quadratic programming problems with fuzzy coefficients.

\section{Introduction}

Quadratic programming is a mathematical modeling technique designed to optimize the usage of limited resources. It has led to a number of interesting applications and the development of numerous useful results (see [1-7]). Quadratic programming is one of the most important optimization techniques in operations research. In real-world applications, quadratic programming models usually are formulated to find some future course of action. The parameter values used would be based on a prediction of future conditions which inevitably involves some degree of uncertainty. If some parameters are imprecise or uncertain, then some crisp values are usually assigned to those uncertain parameters to make the conventional quadratic program workable. The occurrences of randomness and imprecision in the real world are inevitable owing to some unexpected situations. The research on fuzzy mathematical programming has been an active area since Bellman and Zadeh proposed the definition of fuzzy decision [8-12]. As the definition of Bellman and Zadeh, fuzzy decision may be described as the best balance degree of fuzzy objective and resource constraints. In light of the above view, Zimmermann developed a tolerance approach $[10,11]$ for a symmetric model of fuzzy linear programming. Intuitively, when the cost and constraint coefficients and the right-hand sides are fuzzy numbers, the derived objective value is fuzzy as well.

Recently, Liu [13] proposes an effective solution method to solve a class of fuzzy quadratic programming problems. Based on Zadeh's extension principle [11, 14], the fuzzy quadratic programming problem is transformed into a pair of two-level mathematical programs to calculate the upper and lower bounds of the objective value at possibility level $\alpha$. The membership function of the fuzzy objective value is derived numerically by enumerating different values of. MahdaviAmiria and Nasseria $[15,16]$, using a linear ranking function, establish the dual problem of the linear programming problem with trapezoidal fuzzy variables and hence deduce some duality results.

The organization and content of this paper can be summarized as follows. In Section 2, we provide some properties of fuzzy numbers and introduce the concept of fuzzy scalar 
product. Then we provide a discussion of fuzzy numbers and linear ranking functions for ordering them. The definition of the FNQP problem is given and the equivalent quadratic programming of FNQP is presented by utilizing a linear ranking function in Section 3. Section 4 optimality conditions for fuzzy number quadratic programming are presented. We establish duality for the FNQP problem in Section 5 and deduce the duality results. We conclude in Section 6 .

\section{Preliminaries}

2.1. Fuzzy Numbers. We review the fundamental notions of fuzzy set theory, initiated by Bellman and Zadeh [8]. Let $U$ be a universal set. The fuzzy subset $\widetilde{a}$ of $U$ is defined by its membership function $\mu_{\widetilde{a}}: U \rightarrow[0,1]$. The $\alpha$-level set of fuzzy set $\tilde{a}$ is given by

$$
\tilde{a}_{\alpha}= \begin{cases}\left\{x \in U \mid \mu_{\tilde{a}}(x) \geqslant \alpha\right\}, & \text { if } \alpha \in(0,1], \\ \operatorname{cl}\left\{x \in U \mid \mu_{\widetilde{a}}(x)>0\right\}, & \text { if } \alpha=0 .\end{cases}
$$

Definition 1. Let $\widetilde{a}$ be a fuzzy number, that is, a convex normalized fuzzy subset of the real line in the sense that

(a) $\exists x_{0} \in R$ and $\mu_{\tilde{a}}\left(x_{0}\right)=1$, where $\mu_{\tilde{a}}(x)$ is the membership function specifying to what degree $x$ belongs to $\tilde{a}$.

(b) $\mu_{\widetilde{a}}$ is a piecewise continuous function.

The lower and upper bounds of any $\alpha$-level set $\tilde{a}_{\alpha}$ are represented by inf ${ }_{x \in \widetilde{a}_{\alpha}}$ and $\sup _{x \in \widetilde{a}_{\alpha}}$.

A function, usually denoted by " $L$ " or " $R$ ", is a reference function of a fuzzy number if and only if

(1) $L(x)=L(-x)$,

(2) $L(0)=1$,

(3) $L$ is nonincreasing on $[0,+\infty)$.

Definition 2. A convenient representation of fuzzy numbers is in the form of an $L-R$ fuzzy number which is defined as

$$
\mu_{\tilde{a}}(x)= \begin{cases}L\left(\left(a^{L}-x\right) / \alpha\right), & \text { if } x \leqslant a^{L} \text { and } \alpha>0, \\ R\left(\left(x-a^{U}\right) / \beta\right), & \text { if } x \geqslant a^{U} \text { and } \beta>0, \\ 1, & \text { otherwise, }\end{cases}
$$

where $a^{L} \leqslant a^{U},\left[a^{L}, a^{U}\right]$ is the core of $\tilde{a}, \mu_{\tilde{a}}(x)=1, \forall x \in$ $\left[a^{L}, a^{U}\right], a^{L}, a^{U}$ are the lower and upper modal values of $\tilde{a}$, and $\alpha>0, \beta>0$ are the left-hand and right-hand spreads [16].

A flat fuzzy number is denoted by $\tilde{a}=\left[a^{L}, a^{U}, \alpha, \beta\right]_{L R}$. Among the various types of $L-R$ fuzzy numbers, trapezoidal fuzzy numbers, denoted by $\widetilde{a}=\left(a^{L}, a^{U}, \alpha, \beta\right)$, are of the greatest importance. We denote the set of all trapezoidal fuzzy numbers by $\mathscr{F}(\mathbb{R})$. If $a=a^{L}=a^{U}$, then we obtain a triangular fuzzy number, and we show it with $\widetilde{a}=(a, \alpha, \beta)$.
Let $\tilde{a}=\left(a^{L}, a^{U}, \alpha_{1}, \beta_{1}\right)$ and $\tilde{b}=\left(b^{L}, b^{U}, \alpha_{2}, \beta_{2}\right)$, both being trapezoidal fuzzy numbers. We next define arithmetic on the fuzzy numbers $\widetilde{a}$ and $\tilde{b}$ as follows:

$$
\begin{gathered}
x>0, x \in R ; \quad x \cdot \tilde{a}=\left(x a^{L}, x a^{U}, x \alpha_{1}, x \beta_{1}\right), \\
x<0, x \in R ; \quad x \cdot \widetilde{a}=\left(x a^{U}, x a^{L},-x \beta_{1},-x \alpha_{1}\right), \\
\tilde{a}+\tilde{b}=\left(a^{L}+b^{L}, a^{U}+b^{U}, \alpha_{1}+\alpha_{2}, \beta_{1}+\beta_{2}\right), \\
\tilde{a}-\tilde{b}=\left(a^{L}-b^{U}, a^{U}-b^{L}, \alpha_{1}+\beta_{2}, \beta_{1}+\alpha_{2}\right) .
\end{gathered}
$$

2.2. Ranking Functions. There are different methods for comparison of fuzzy numbers [17-22]. One of the most convenient methods is comparison by use of ranking functions [23, 24]. In fact, an efficient approach for ordering the elements of $\mathscr{F}(R)$ is to define a ranking function $\mathscr{A}$ : $\mathscr{F}(R) \longrightarrow R$ which maps each fuzzy number into the real line, where a natural order exists. We define orders on $\mathscr{F}(R)$ by

$$
\begin{aligned}
& \widetilde{a} \geqslant_{\mathscr{A}} \widetilde{b} \quad \text { if and only if } \mathscr{A}(\widetilde{a}) \geqslant \mathscr{A}(\widetilde{b}), \\
& \widetilde{a}>_{\mathscr{A}} \widetilde{b} \quad \text { if and only if } \mathscr{A}(\widetilde{a})>\mathscr{A}(\widetilde{b}), \\
& \widetilde{a}=_{\mathscr{A}} \widetilde{b} \quad \text { if and only if } \mathscr{A}(\widetilde{a})=\mathscr{A}(\widetilde{b}),
\end{aligned}
$$

where $\widetilde{a}, \widetilde{b} \in \mathscr{F}(R)$. Also we write $\widetilde{a} \leqslant_{\mathscr{A}} \widetilde{b}$ if and only if $\tilde{b} \geqslant \mathscr{a}$.

We restrict our attention to linear ranking functions, that is, a ranking function $\mathscr{A}$ such that

$$
\mathscr{A}(k \widetilde{a}+\widetilde{b})=k \mathscr{A}(\widetilde{a})+\mathscr{A}(\widetilde{b}),
$$

for all $\tilde{a}, \tilde{b} \in \mathscr{F}(R)$ and $k \in R$. The following lemma is now immediate.

Lemma 3 (see [16]). Let $\mathscr{A}$ be any linear ranking function and, without loss of generality, let $\widetilde{0}=(0,0,0,0)$; then

(i) $\tilde{a} \geqslant_{\mathscr{A}} \widetilde{b}$ if and only if $\widetilde{a}-\widetilde{b} \geqslant_{\mathscr{A}} \tilde{0}$ if and only if $-\widetilde{a} \leqslant_{\mathscr{A}}-\widetilde{b}$.

(ii) If $\tilde{a} \geqslant_{\mathscr{A}} \tilde{b}$ and $\tilde{c} \geqslant_{\mathscr{A}} \tilde{d}$, then $\tilde{a}+\tilde{c} \geqslant_{\mathscr{A}} \tilde{b}+\tilde{d}$.

(iii) Let $\tilde{a} \geqslant_{\mathscr{A}} \tilde{b}$; if $x>0$, then $x \widetilde{a} \geqslant_{\mathscr{A}} x \tilde{b}$; otherwise, $x \tilde{a} \leqslant_{\mathscr{A}} x \tilde{b}$.

Here, we introduce a linear ranking function that is similar to the ranking function adopted by Maleki et al. [24]. For any $L-R$ fuzzy number $\tilde{a}=\left[a^{L}, a^{U}, \alpha, \beta\right]_{L R}$, we use ranking function as follows:

$$
\mathscr{A}(\widetilde{a})=\int_{0}^{1}\left(R_{\tilde{a}}^{-1}(\lambda)+L_{\widetilde{a}}^{-1}(\lambda)\right) d \lambda,
$$

where $\lambda \in[0,1]$ and $R_{\widetilde{a}}^{-1}$ and $L_{\widetilde{a}}^{-1}$ are inverse function of $R_{\widetilde{a}}$ and $L_{\tilde{a}}$, respectively. In the case of trapezoidal fuzzy numbers $\tilde{a}=\left(a^{L}, a^{U}, \gamma, \delta\right)$, we have

$$
\mathscr{A}(\widetilde{a})=\int_{0}^{1}\left(\inf _{x \in \widetilde{a}_{\alpha}}+\sup _{x \in \widetilde{A}_{\alpha}}\right) d \alpha,
$$


where $\tilde{a}_{\alpha}$ is the $\alpha$-level set of $\widetilde{a}$. Then, for trapezoidal fuzzy numbers $\widetilde{a}=\left(a^{L}, a^{U}, \alpha_{1}, \beta_{1}\right)$ and $\widetilde{b}=\left(b^{L}, b^{U}, \alpha_{2}, \beta_{2}\right)$, we can get

$$
\begin{gathered}
\mathscr{A}(\widetilde{a})=a^{L}+a^{U}+\frac{1}{2}\left(\beta_{1}-\alpha_{1}\right), \\
\widetilde{a} \geqslant \mathscr{A} \widetilde{b} \\
i f f a^{L}+a^{U}+\frac{1}{2}\left(\beta_{1}-\alpha_{1}\right) \geqslant b^{L}+b^{U}+\frac{1}{2}\left(\beta_{2}-\alpha_{2}\right) .
\end{gathered}
$$

Definition 4. We shall say that the real number $r$ corresponds to the fuzzy number $\widetilde{r}$, with respect to a given linear ranking function $\mathscr{A}$, if $r=\mathscr{A}(\widetilde{r})$.

\section{Fuzzy Number Quadratic Programming Problems}

In this section, we first define fuzzy number quadratic programming problems with fuzzy coefficients. Then, using ranking functions for comparison of fuzzy numbers, we define a crisp model which is equivalent to the fuzzy quadratic programming problem with fuzzy coefficients and use optimal solution of this model as the optimal solution of fuzzy number quadratic programming problem with fuzzy number coefficients.

Definition 5. Let $\mathscr{F}(R)$ be the set of all trapezoidal fuzzy numbers. The model

$$
\begin{aligned}
& \min \quad \tilde{z}=\mathscr{A} \widetilde{c}^{T} x+\frac{1}{2} x^{T} \widetilde{Q} x, \\
& \text { s.t. } \widetilde{A} x \leqslant \mathscr{A} \widetilde{b}, \\
& x \in R^{n} \text {, }
\end{aligned}
$$

or

$$
\begin{array}{ll}
\min \quad & \tilde{z}=_{\mathscr{A}} \sum_{j=1}^{n} \widetilde{c}_{j} x_{j}+\frac{1}{2} \sum_{k=1}^{m} \sum_{j=1}^{n} \widetilde{q}_{k j} x_{k} x_{j}, \\
\text { s.t. } & \sum_{j=1}^{n} \widetilde{a}_{i j} x_{j} \leqslant \mathscr{A} \widetilde{b}_{i}, \quad i=1,2, \ldots, m, \\
& x_{j} \in R, \quad j=1,2, \ldots, n,
\end{array}
$$

where $\widetilde{Q}=\left(\widetilde{q}_{k j}\right)_{n \times n}, \widetilde{A}=\left(\widetilde{a}_{i j}\right)_{m \times n}, \widetilde{c}=\left(\widetilde{c}_{1}, \widetilde{c}_{2}, \ldots, \widetilde{c}_{n}\right)^{T}, \widetilde{b}=$ $\left(\widetilde{b}_{1}, \widetilde{b}_{2}, \ldots, \widetilde{b}_{m}\right)^{T}$, and $\widetilde{q}_{k j}, \widetilde{a}_{i j}, \widetilde{c}_{j}, \widetilde{b}_{i} \in \mathscr{F}(R)$, for $i=$ $1,2, \ldots, m, k=1,2, \ldots, n, j=1,2, \ldots, n$, is called a fuzzy number quadratic programming model.

Definition 6. Any $x$ which satisfies the set of constraints of FNQP is called a feasible solution. Let $\mathbb{F}$ be the set of all feasible solutions of FNQP. We shall say that $x_{0} \in \mathbb{F}$ is a global optimal feasible solution for FNQP if $\widetilde{c}^{T} x+$ $(1 / 2) x^{T} \widetilde{Q} x \leqslant{ }_{\mathscr{A}} \widetilde{c}^{T} x_{0}+(1 / 2) x_{0}^{T} \widetilde{\mathrm{Q}} x_{0}$ for any $x \in \mathbb{F}$. We say that $x_{0} \in \mathbb{F}$ is a local optimal feasible solution for
FNQP if there exists a neighborhood $U$ of $x_{0}$ such that $\tilde{c}^{T} x+$ $(1 / 2) x^{T} \widetilde{Q} x \leqslant \mathscr{A}^{T} x_{0}+(1 / 2) x_{0}^{T} \widetilde{Q} x_{0}$ for all $x \in U \bigcap \mathbb{F}$.

The following theorem shows that any FNQP can be reduced to a quadratic programming problem.

Theorem 7. The following quadratic programming problem $(Q P P)$ and the FNQP in (9) are equivalent:

$$
\begin{aligned}
& \min \quad z=c^{T} x+\frac{1}{2} x^{T} Q x, \\
& \text { s.t. } \quad A x \leqslant b, \\
& \quad x \in R^{n},
\end{aligned}
$$

or

$$
\begin{array}{ll}
\min & z=\sum_{j=1}^{n} c_{j} x_{j}+\frac{1}{2} \sum_{k=1}^{m} \sum_{j=1}^{n} q_{k j} x_{k} x_{j}, \\
\text { s.t. } & \sum_{j=1}^{n} a_{i j} x_{j} \leqslant b_{i}, \quad i=1,2, \ldots, m, \\
& x_{j} \in R, \quad j=1,2, \ldots, n,
\end{array}
$$

where $=\left(q_{k j}\right)_{n \times n}, A=\left(a_{i j}\right)_{m \times n}$, and $q_{k j}, a_{i j}, b_{i}, c_{j}$ are real numbers corresponding to the fuzzy numbers $\widetilde{q}_{k j}, \widetilde{a}_{i j}, \widetilde{b}_{i}, \widetilde{c}_{j}$ with respect to a given linear ranking function $\mathscr{A}$, respectively.

Proof. The method of proof is the same as Lemma 3.1 in [24]. However, to provide a self-contained presentation, and because this result is central to this paper, we provide a direct proof. Let $\mathbb{F}_{1}$ and $\mathbb{F}_{2}$ be the set of all feasible solutions of (9) and (11), respectively. We first prove that $\mathbb{F}_{1}=\mathbb{F}_{2}$. We see

$$
\begin{aligned}
x \in \mathbb{F}_{1} \Longleftrightarrow \sum_{j=1}^{n} \tilde{a}_{i j} x_{j} \leqslant \mathscr{A} \tilde{b}_{i}, \quad i=1,2, \ldots, m, \\
\Longleftrightarrow \sum_{j=1}^{n}\left(a_{i j}^{L}, a_{i j}^{U}, \alpha_{i j}, \beta_{i j}\right) x_{j} \\
\quad \leqslant \mathscr{A}\left(b_{i}^{L}, b_{i}^{U}, \alpha_{i}, \beta_{i}\right), \quad i=1,2, \ldots, m, \\
\Longleftrightarrow \mathscr{A}\left[\sum_{j=1}^{n}\left(a_{i j}^{L}, a_{i j}^{U}, \alpha_{i j}, \beta_{i j}\right) x_{j}\right] \\
\quad \leqslant \mathscr{A}\left[\left(b_{i}^{L}, b_{i}^{U}, \alpha_{i}, \beta_{i}\right)\right], \quad i=1,2, \ldots, m, \\
\Longleftrightarrow \sum_{j=1}^{n}\left(\mathscr{A}\left[a_{i j}^{L}, a_{i j}^{U}, \alpha_{i j}, \beta_{i j}\right]\right) x_{j} \\
\quad \leqslant \mathscr{A}\left[\left(b_{i}^{L}, b_{i}^{U}, \alpha_{i}, \beta_{i}\right)\right], \quad i=1,2, \ldots, m, \\
\Longleftrightarrow \sum_{j=1}^{n} a_{i j} x_{j} \leqslant b_{i}, \quad i=1,2, \ldots, m, \\
\Longleftrightarrow x \in \mathbb{F}_{2} .
\end{aligned}
$$


Then, we have $\mathbb{F}_{1}=\mathbb{F}_{2}$. Let $x^{0}$ be an optimal feasible solution for (9); then, for all $x \in \mathbb{F}_{1}$, we have

$$
\begin{aligned}
& \widetilde{c}^{T} x+\frac{1}{2} x^{T} \widetilde{Q} x \geqslant_{\mathscr{A}} \widetilde{c}^{T} x^{0}+\frac{1}{2}\left(x^{0}\right)^{T} \widetilde{Q} x^{0}, \\
& \Longleftrightarrow \sum_{j=1}^{n} \widetilde{c}_{j} x_{j}+\frac{1}{2} \sum_{k=1}^{m} \sum_{j=1}^{n} \widetilde{q}_{k j} x_{k} x_{j} \\
& \geqslant_{\mathscr{A}} \sum_{j=1}^{n} \widetilde{c}_{j} x_{j}^{0}+\frac{1}{2} \sum_{k=1}^{m} \sum_{j=1}^{n} \widetilde{q}_{k j} x_{k}^{0} x_{j}^{0}, \\
& \Longleftrightarrow \mathscr{A}\left(\sum_{j=1}^{n} \widetilde{c}_{j} x_{j}+\frac{1}{2} \sum_{k=1}^{m} \sum_{j=1}^{n} \widetilde{q}_{k j} x_{k} x_{j}\right) \\
& \geqslant \mathscr{A}\left(\sum_{j=1}^{n} \widetilde{c}_{j} x_{j}^{0}+\frac{1}{2} \sum_{k=1}^{m} \sum_{j=1}^{n} \widetilde{q}_{k j} x_{k}^{0} x_{j}^{0}\right), \\
& \Longleftrightarrow \sum_{j=1}^{n} \mathscr{A}\left(\widetilde{c}_{j}\right) x_{j}+\frac{1}{2} \sum_{k=1}^{m} \sum_{j=1}^{n} \mathscr{A}\left(\widetilde{q}_{k j}\right) x_{k} x_{j} \\
& \geqslant \sum_{j=1}^{n} \mathscr{A}\left(\tilde{c}_{j}\right) x_{j}^{0}+\frac{1}{2} \sum_{k=1}^{m} \sum_{j=1}^{n} \mathscr{A}\left(\tilde{q}_{k j}\right) x_{k}^{0} x_{j}^{0}, \\
& \Longleftrightarrow \sum_{j=1}^{n} c_{j} x_{j}+\frac{1}{2} \sum_{k=1}^{m} \sum_{j=1}^{n} q_{k j} x_{k} x_{j} \\
& \geqslant \sum_{j=1}^{n} c_{j} x_{j}^{0}+\frac{1}{2} \sum_{k=1}^{m} \sum_{j=1}^{n} q_{k j} x_{k}^{0} x_{j}^{0} .
\end{aligned}
$$

So, $x^{0}$ is an optimal feasible solution for (11) or (12).

Now, let $x^{0}$ be an optimal feasible solution for (11) or (12). We shall assume that $x^{0}$ is not an optimal feasible solution for (9) and exhibit a contradiction. If $x^{0}$ is not an optimal feasible solution for (9), then there exists a $x^{1} \in \mathbb{F}_{1}$ such that

$$
\tilde{c}^{T} x^{0}+\frac{1}{2}\left(x^{0}\right)^{T} \widetilde{Q} x^{0}>_{\mathscr{A}} \tilde{c}^{T} x^{1}+\frac{1}{2}\left(x^{1}\right)^{T} \widetilde{Q} x^{1} .
$$

From the ranking function (8), (15) can be written as

$$
c^{T} x^{0}+\frac{1}{2}\left(x^{0}\right)^{T} \mathrm{Q} x^{0}>c^{T} x^{1}+\frac{1}{2}\left(x^{1}\right)^{T} \mathrm{Q} x^{1},
$$

which contradicts the facts that $x^{0}$ is an optimal feasible solution for (11), since $x^{1} \in \mathbb{F}_{2}$.

From Theorem 7, we have that the sets of all feasible solutions of FNQP and QPP are the same. And if $x^{*}$ is an optimal feasible solution for FNQP, then $x^{*}$ is an optimal feasible solution for QPP. Then we can easily see the following result.

Corollary 8. If QPP does not have a solution, then FNQP does not have a solution either.

Definition 9. $\widetilde{Q}=\left(\widetilde{q}_{k j}\right)_{n \times n}$ is denoted by a symmetric fuzzy number matrix if $\widetilde{q}_{k j} \in \mathscr{F}(R)$ and $\widetilde{q}_{k j}=\widetilde{q}_{j k}$ for any $k, j=$ $1,2, \ldots, n$.
Let $Q=\left(q_{k j}\right)_{n \times n}$ be a real symmetric matrix corresponding to the symmetric fuzzy numbers matrix $\widetilde{Q}=\left(\widetilde{q}_{k j}\right)_{n \times n}$ and $Q=\mathscr{A}(\widetilde{Q})$. In the following, we only consider the symmetric fuzzy numbers matrix $\widetilde{Q}=\left(\widetilde{q}_{k j}\right)_{n \times n}$.

Definition 10. Let $\widetilde{Q}$ be a symmetric fuzzy numbers matrix and $Q=\mathscr{A}(\widetilde{Q})$ by the ranking function (8).

(i) $\widetilde{Q}$ is called a fuzzy number positive definite matrix (resp., fuzzy number negative definite matrix) if $x^{T} \widetilde{Q} x>_{\mathscr{A}} 0$ (resp., $x^{T} \widetilde{Q} x<{ }_{\mathscr{A}} 0$ ) for all nonzero $x$ in $R^{n}$ or $Q$ is a positive definite matrix (resp., negative definite matrix).

(ii) If $x^{T} \widetilde{Q} x \geqslant_{\mathscr{A}} 0 \quad$ (resp., $\left.x^{T} \widetilde{Q} x \leqslant{ }_{A} 0\right)$ for all $x$ in $R^{n}$, or $Q$ is a positive semidefinite matrix (resp., negative semidefinite matrix), then $\widetilde{Q}$ is said to be a fuzzy number positive semidefinite (resp., fuzzy number negative semidefinite).

(iii) If $Q$ is an indefinite matrix, then $\widetilde{Q}$ is said to be a fuzzy number indefinite matrix.

Definition 11. If the fuzzy number matrix $\widetilde{Q}$ is fuzzy number positive semidefinite matrix, then (9) is called a convex fuzzy number quadratic programming problem. If $\widetilde{Q}$ is fuzzy number positive definite matrix, (9) is a strict convex FNQP. Equation (9) is said to be a nonconvex FNQP if $\widetilde{Q}$ is fuzzy number indefinite matrix.

From Theorem 7 and definition above, the following result is now immediate.

Corollary 12. (i) If $\widetilde{Q}$ is fuzzy number positive semidefinite matrix, then the local solution $x^{*}$ of (9) is a global solution.

(ii) If $\widetilde{Q}$ is fuzzy number positive definite matrix, then the local solution $x^{*}$ of (9) is a unique global solution.

Example 13. Consider the following FNQP:

$$
\begin{aligned}
& \min \quad \widetilde{z}=\frac{1}{2}(1,3,2,2) x_{1}^{2} \\
&+\frac{1}{2}(0.5,1.5,1,1) x_{2}^{2}-(1,1,4,2) x_{1} x_{2} \\
& \text { s.t. } \quad(2,3,2,2) x_{1}+(1,3,2,3) x_{2} \leqslant \mathscr{A}(5,6,2,2), \\
& \quad(2,3,1,3) x_{1}+(1,2,3,1) x_{2} \leqslant \mathscr{A}(3,5,2,4), \\
& \quad-(-1,2,1,1) x_{1}-(0,2,4,2) x_{2} \leqslant \mathscr{A}-(0,1,1,1),
\end{aligned}
$$

where coefficients are trapezoidal fuzzy numbers.

We apply the ranking function (8) to solve the above FNQP. The problem reduces to

$$
\begin{array}{ll}
\min & z=2 x_{1}^{2}+x_{2}^{2}-x_{1} x_{2} \\
\text { s.t. } & 5 x_{1}+4.5 x_{2} \leqslant 11, \\
& 6 x_{1}+2 x_{2} \leqslant 9, \\
& -x_{1}-x_{2} \leqslant-1 .
\end{array}
$$


Then we see that coefficient matrix of (18) is $Q=\left(\begin{array}{cc}4 & -1 \\ -1 & 2\end{array}\right)$. Since $Q$ is a positive definite matrix, (17) is a strict convex FNQP and the local solution $x^{*}$ of (18) is a global solution of (17).

The optimal solution of (18) is $x_{1}^{*}=0.375, x_{2}^{*}=0.625$; then the optimal solution of (17) is $x_{1}^{*}=0.375, x_{2}^{*}=$ 0.625 , and the optimal objective value is $\widetilde{z}^{*}=(-0.06640625$, $0.26953125,0.8046875,1.2734375)$. So, by the ranking function (8), $\mathscr{A}\left(\widetilde{z}^{*}\right)=0.4375$.

\section{Optimality Conditions for Fuzzy Number Quadratic Programming}

Let us now state the necessary optimality conditions of problem (9).

Theorem 14 (necessary conditions). Let $x^{*}$ be a local minimizer of the fuzzy number quadratic programming problem (9). Then there exists a m-vector $\lambda^{*} \in R^{m}$ such that

$$
\begin{gathered}
\tilde{c}+\widetilde{Q} x^{*}-\widetilde{A} \lambda^{*}=\mathscr{0}, \\
\lambda_{i}^{*}\left[\left(\widetilde{a}^{i}\right) x^{*}-\widetilde{b}_{i}\right]={ }_{\mathscr{A}} \widetilde{0}, \quad i=1,2, \ldots, m, \\
\lambda^{*} \geqslant 0,
\end{gathered}
$$

where $\widetilde{a}^{i}$ is the ith row of $m \times n$ fuzzy number matrix $\widetilde{A}$, and, for every $d \neq 0$, satisfying

$$
\tilde{a}^{i} d={ }_{\mathscr{A}} \widetilde{0}, \quad i \in I\left(x^{*}\right),
$$

where

$$
I\left(x^{*}\right)=\left\{i \mid \tilde{a}^{i} x^{*}={ }_{\mathscr{A}} \tilde{b}_{i}, i=1,2, \ldots, m\right\},
$$

we have

$$
d^{T} \widetilde{Q} d \geqslant_{\mathscr{A}} \widetilde{0} .
$$

Proof. Since $x^{*}$ is a local minimizer of fuzzy number quadratic programming problem (9), $x^{*}$ is a local minimizer of quadratic programming problem (11) by Theorem 7 . Then, from Theorem 7.1 in Avriel [25], there exists a $m$-vector $\lambda^{*} \epsilon$ $R^{m}$ such that

$$
\begin{gathered}
c+Q x^{*}-A \lambda^{*}=0, \\
\lambda_{i}^{*}\left[\left(a^{i}\right) x^{*}-b_{i}\right]=0, \quad i=1,2, \ldots, m, \\
\lambda^{*} \geq 0,
\end{gathered}
$$

where $c=\mathscr{A}(\widetilde{c}), Q=\mathscr{A}(\widetilde{Q}), A=\mathscr{A}(\widetilde{A}), b_{i}=\mathscr{A}\left(\widetilde{b}_{i}\right)$ for any $i=1,2, \ldots, m$, and $a^{i}$ is the $i$ th row of $m \times n$ matrix $A$, and, for every $d \neq 0$, satisfying

$$
a^{i} d=0, \quad i \in I\left(x^{*}\right),
$$

where

$$
I\left(x^{*}\right)=\left\{i \mid a^{i} x^{*}=b_{i}, i=1,2, \ldots, m\right\},
$$

we have

$$
d^{T} Q d \geqslant 0 .
$$

From the ranking function (8), formulas (19)-(22) and (23)-(26) are equivalent and the proof is complete.

Turning to sufficient conditions, we first define

$$
\widehat{I}\left(x^{*}\right)=\left\{i: i \in I\left(x^{*}\right), \lambda^{*}>0\right\} .
$$

Let us now state the sufficient optimality conditions for problem (9).

Theorem 15 (sufficient conditions). Let $x^{*}$ be feasible for problem (9). If there exists a vector $\lambda^{*} \in R^{m}$ satisfying (19)(22), and for every $0 \neq b \in R^{m}$ such that

$$
\begin{gathered}
\tilde{a}^{i} b=\mathscr{A}_{\mathcal{D}} \widetilde{0}, \quad i \in \widehat{I}\left(x^{*}\right), \\
\tilde{a}^{i} b \leqslant_{\mathscr{A}} \widetilde{0}, \quad i \in I\left(x^{*}\right), i \notin \widehat{I}\left(x^{*}\right),
\end{gathered}
$$

it follows that

$$
b^{T} \widetilde{Q} b>_{\mathscr{A}} \widetilde{0}
$$

then $x^{*}$ is a strict local minimum of FNQP.

Proof. From the ranking function (8), there exists a vector $\lambda^{*} \in R^{m}$ satisfying (23)-(26), and for every $0 \neq b \in R^{m}$ such that

$$
\begin{gathered}
a^{i} b=0, \quad i \in \widehat{I}\left(x^{*}\right), \\
a^{i} b \leqslant 0, \quad i \in I\left(x^{*}\right), \quad i \notin \widehat{I}\left(x^{*}\right),
\end{gathered}
$$

it follows that

$$
b^{T} Q b>0 .
$$

Then, from Theorem 7.2 in Avriel [25], $x^{*}$ is a strict local minimum of (11). So $x^{*}$ is a strict local minimum of FNQP by Theorem 7 .

Next, we give a sufficient and necessary optimality condition for FNQP (9).

Theorem 16 (necessary and sufficient conditions). Let $x^{*}$ be feasible for problem (9); then $x^{*}$ is a local minimizer if and only if there exists $\left(x^{*}, \lambda^{*}\right)$ such that (19)-(22) hold, and

$$
d^{T} \widetilde{\mathrm{Q}} d \geqslant_{\mathscr{A}} \widetilde{0}, \quad \forall d \in \widetilde{\mathrm{Q}}\left(x^{*}, \lambda^{*}\right),
$$

where

$$
\begin{gathered}
\widetilde{Q}\left(x^{*}, \lambda^{*}\right)=\left\{d \neq 0 \mid \tilde{a}^{i} d \leqslant_{\mathscr{A}} \widetilde{0}, i \in I\left(x^{*}\right),\right. \\
\tilde{a}^{i} d=\mathscr{A}, i \in I\left(x^{*}\right), \\
\left.\lambda^{*}>0\right\} .
\end{gathered}
$$


Proof. Using the ranking function (8), formulas (19)-(22) and formulas (23)-(26) are equivalent. Then (32) and (33) are respectively equivalent to the following formulas:

$$
d^{T} Q d \geqslant 0, \quad \forall d \in Q\left(x^{*}, \lambda^{*}\right),
$$

where

$$
\begin{aligned}
& Q\left(x^{*}, \lambda^{*}\right)=\left\{d \neq 0 \mid a^{i} d \leqslant 0, i \in I\left(x^{*}\right),\right. \\
& \left.a^{i} d=0, i \in I\left(x^{*}\right), \lambda^{*}>0\right\} .
\end{aligned}
$$

By Theorem 3.4 in Lee et al. [26], $x^{*}$ is a local minimizer of (11) if and only if there exists $\left(x^{*}, \lambda^{*}\right)$ such that (23)-(26) and (34)-(35) hold. Then, based on Theorem 7, we complete the proof.

\section{Duality for Fuzzy Number Quadratic Programming}

Similar to the duality theory in quadratic programming (see, e.g., Mangasarian [27]), for every FNQP, there is an associated problem which satisfies some important properties. We shall call this related FNQP the dual fuzzy number quadratic programming (DFNQP).

\subsection{Dual Problem. For the FNQP}

$$
\begin{array}{ll}
\min & \widetilde{z}={ }_{\mathscr{A}} \widetilde{c}^{T} x+\frac{1}{2} x^{T} \widetilde{Q} x, \\
\text { s.t. } & \widetilde{A} x \leqslant_{\mathscr{A}} \widetilde{b}, \\
& x \in R^{n},
\end{array}
$$

define the dual fuzzy number quadratic programming problem (DFNQP) as

$$
\begin{array}{ll}
\max & \widetilde{y}={ }_{\mathscr{A}}-\widetilde{b}^{T} u-\frac{1}{2} x^{T} \widetilde{\mathrm{Q}} x, \\
\text { s.t. } & \widetilde{\mathrm{Q}} x+\widetilde{A}^{T} u=_{\mathscr{A}} \widetilde{\mathcal{c}}, \\
& u \geqslant 0, \quad x \in R^{n}, \quad u \in R^{m} .
\end{array}
$$

By the ranking function (8), problem (37) and the following quadratic programming problem are equivalent:

$$
\begin{array}{ll}
\max & y=-b^{T} u-\frac{1}{2} x^{T} Q x, \\
\text { s.t. } & Q x+A^{T} u=c, \\
& u \geqslant 0, \quad x \in R^{n}, \quad u \in R^{m} .
\end{array}
$$

We see that (38) is the dual quadratic programming problem for (9) (see, e.g., Mangasarian [27]).
Example 17. Consider the given FNQP in Example 13. The dual to this problem follows:

$$
\begin{array}{ll}
\max \quad \tilde{y}=\mathscr{A} & -\frac{1}{2}(1,3,2,2) x_{1}^{2} \\
& -\frac{1}{2}(0.5,1.5,1,1) x_{2}^{2}+(1,1,4,2) x_{1} x_{2} \\
& -(5,6,2,2) u_{1}-(3,5,2,4) u_{2}+(0,1,1,1) u_{3}, \\
\text { s.t. } \quad(1,3,2,2) x_{1}-(1,1,4,2) x_{2}+(2,3,2,2) u_{1} \\
+(2,3,1,3) u_{2}-(-1,2,1,1) u_{3}={ }_{\mathscr{A}} 0, \\
-(1,1,4,2) x_{1}+(0.5,1.5,1,1) x_{2}(1,3,2,3) u_{1} \\
+(1,2,3,1) u_{2}-(0,2,4,2) u_{3}={ }_{\mathscr{A}} 0, \\
u_{1}, u_{2} \geqslant 0, \quad x_{1}, x_{2} \in R .
\end{array}
$$

Now if we apply the ranking function (8), we have

$$
\begin{array}{ll}
\max & y=-2 x_{1}^{2}-x_{2}^{2}+x_{1} x_{2}-11 u_{1}-9 u_{2}+u_{3} \\
\text { s.t. } & 4 x_{1}-x_{2}+5 u_{1}+6 u_{2}-u_{3}=0, \\
& -x_{1}+2 x_{2}+4.5 u_{1}+2 u_{2}-u_{3}=0 \\
& u_{1}, u_{2} \geqslant 0, \quad x_{1}, \quad x_{2} \in R .
\end{array}
$$

The optimal solution is $x_{1}^{*}=0.375, x_{2}^{*}=0.625, u_{1}=$ $0, u_{2}=0, u_{3}=0.875$, and the optimal objective value is $\tilde{y}^{*}=(-0.26953125,0.94140625,2.15625,1.6875)$. So, by the ranking function $(8), \mathscr{A}\left(\tilde{y}^{*}\right)=0.4375$.

5.2. The Relationships between FNQP and DFNQP. We shall discuss here the relationships between the fuzzy number quadratic programming problem and its corresponding dual. Let $\mathbb{F}_{1}$ and $\mathbb{F}_{2}$ be the feasible solution sets of FNQP and DFNQP, respectively.

Lemma 18. Dual of DFNP is FNQP.

Proof. Use Lemma 3 and the definition of DFNQP.

Lemma 18 indicates that the duality results can be applied to any of the primal or dual problems posed as the primal problem.

Theorem 19 (weak duality theorem). Let $\widetilde{Q}$ be fuzzy number positive semidefinite matrix, $x^{0} \in \mathbb{F}_{1},\left(x^{1}, u^{1}\right) \in \mathbb{F}_{2}$; then we have

$$
-\widetilde{b}^{T} u^{1}-\frac{1}{2}\left(x^{1}\right)^{T} \widetilde{Q} x^{1} \leqslant_{\mathscr{A}} \widetilde{c}^{T} x^{0}+\frac{1}{2}\left(x^{0}\right)^{T} \widetilde{Q} x^{0} .
$$

Proof. By the ranking function (8), (41) holds if and only if

$$
-b^{T} u^{1}-\frac{1}{2}\left(x^{1}\right)^{T} Q x^{1} \leqslant c^{T} x^{0}+\frac{1}{2}\left(x^{0}\right)^{T} Q x^{0},
$$

where $b, Q, c$ are real numbers corresponding to the fuzzy numbers $\widetilde{b}, \widetilde{Q}, \widetilde{c}$. Equation (42) follows from Theorem 8.1.3 
in [27] by observing that $c^{T} x+(1 / 2)(x)^{T} Q x$ is convex on $R^{n}$ if the matrix $Q$ is positive semidefinite.

The following corollaries are immediate consequences of Theorem 19.

Corollary 20. Let $\widetilde{Q}$ be fuzzy number positive semidefinite matrix. If $x^{0}$ and $\left(x^{1}, u^{1}\right)$ are feasible solutions to FNQP and $D F N Q P$, respectively, and $-\widetilde{b}^{T} u^{1}-(1 / 2)\left(x^{1}\right)^{T} \widetilde{Q} x^{1}={ }_{\mathscr{A}} \widetilde{c}^{T} x^{0}+$ $(1 / 2)\left(x^{0}\right)^{T} \widetilde{Q} x^{0}$, then $x^{0}$ and $\left(x^{1}, u^{1}\right)$ are optimal solutions to their respective problems.

Definition 21. We say that FNLPP (or DFNLPP) is unbounded if feasible solutions exist with arbitrary small (or large) ranking values for the fuzzy objective function.

Corollary 22. If either problem is unbounded, then the other problem has no feasible solution.

Theorem 23. Let $\widetilde{Q}$ be fuzzy number positive semidefinite matrix. If $x^{*}$ solves FNQP (36), then $x^{*}$ and some $u^{*} \in R^{m}$ solves DFNQP (37) and the two optimal values of ranking functions for the fuzzy objectives are equal.

Proof. Since $x^{*}$ solves FNQP (36), then $x^{*}$ is the optimal solutions to QPP (11). From Dorn's duality theorem [27], there exist $x^{*}$ and some $u^{*} \in R^{m}$ solves problem (38) since $Q$ is positive semidefinite matrix, and $-b^{T} u^{*}-(1 / 2)\left(x^{*}\right)^{T} Q x^{*}=$ $C^{T} x^{*}+(1 / 2)\left(x^{*}\right)^{T} Q x^{*}$. Then, $\left(x^{*}, u^{*}\right)$ solves DFNQP (37) and $-\widetilde{b}^{T} u^{*}-(1 / 2)\left(x^{*}\right)^{T} \widetilde{Q} x^{*}=\mathscr{A}_{\mathscr{A}} \widetilde{c}^{T} x^{*}+(1 / 2)\left(x^{*}\right)^{T} \widetilde{Q} x^{*}$. For if $-\widetilde{b}^{T} u^{*}-(1 / 2)\left(x^{*}\right)^{T} \widetilde{Q} x^{*} \neq \mathscr{A}_{\mathscr{A}} \widetilde{c}^{T} x^{*}+(1 / 2)\left(x^{*}\right)^{T} \widetilde{\mathrm{Q}} x^{*}$, then, by the ranking function $(8),-b^{T} u^{*}-(1 / 2)\left(x^{*}\right)^{T} Q x^{*} \neq C^{T} x^{*}+$ $(1 / 2)\left(x^{*}\right)^{T} Q x^{*}$, which contradicts $-b^{T} u^{*}-(1 / 2)\left(x^{*}\right)^{T} Q x^{*}=$ $C^{T} x^{*}+(1 / 2)\left(x^{*}\right)^{T} Q x^{*}$.

For an illustration of the above theorem, consider the FNQP and DFNQP given in Examples 13 and 17, respectively. Symmetric fuzzy number matrix $\widetilde{Q}=\left(\begin{array}{cc}(1,3,2,2) & -(1,1,4,2) \\ -(1,1,4,2) & (0.5,1,5,1,1)\end{array}\right)$ is fuzzy number positive definite, since matrix $Q=\mathscr{A}(\widetilde{Q})=$ $\left(\begin{array}{cc}4 & -1 \\ -1 & 2\end{array}\right)$ is positive definite. We see that the optimal solution for the FNQP is $x^{*}=(0.375,0.626)$; then there exists $u_{1}=$ $0, u_{2}=0, u_{3}=0.875$ such that $x_{1}^{*}=0.375, x_{2}^{*}=0.625, u_{1}=$ $0, u_{2}=0, u_{3}=0.875$ solves DFNQP and $\widetilde{z}^{*}=\mathscr{A} \tilde{y}^{*}$ by the ranking function (8).

Theorem 24 (strict converse duality theorem). Let $\widetilde{\mathrm{Q}}$ be fuzzy number positive definite matrix. If $\left(x^{*}, u^{*}\right)$ is an optimal solution to DFNQP (37), then $x^{*}$ solves FNQP (36), and $-\widetilde{b}^{T} u^{*}-(1 / 2)\left(x^{*}\right)^{T} \widetilde{Q} x^{*}={ }_{\mathscr{A}} \widetilde{c}^{T} x^{*}+(1 / 2)\left(x^{*}\right)^{T} \widetilde{Q} x^{*}$.

Proof. Since $\widetilde{Q}$ is fuzzy number positive definite and $\left(x^{*}, u^{*}\right)$ is an optimal solution to DFNQP (37), we have that $Q$ is positive definite and $\left(x^{*}, u^{*}\right)$ is an optimal solution to problem (38). From Theorem 8.2.5 in [27], $x^{*}$ solves QPP (11) and $-b^{T} u^{*}-(1 / 2)\left(x^{*}\right)^{T} Q x^{*}=C^{T} x^{*}+(1 / 2)\left(x^{*}\right)^{T} Q x^{*}$. From Theorem 7 above, then $x^{*}$ solves FNQP (36), and $-\tilde{b}^{T} u^{*}-$ $(1 / 2)\left(x^{*}\right)^{T} \widetilde{\mathrm{Q}} x^{*}={ }_{\mathscr{A}} \tilde{c}^{T} x^{*}+(1 / 2)\left(x^{*}\right)^{T} \widetilde{\mathrm{Q}} x^{*}$.
Theorem 25 (Dorn's converse duality theorem). Let $\widetilde{Q}$ be fuzzy number positive semidefinite matrix. If $\left(x^{*}, u^{*}\right)$ solves $D F N Q P$ (37), then some $\bar{x} \in R^{n}$ (not necessarily equal to $x^{*}$ ), satifying $\widetilde{Q}\left(\bar{x}-x^{*}\right)={ }_{\mathscr{A}} 0$, solves FNQP (36), and $-\widetilde{b}^{T} u^{*}-$ $(1 / 2)\left(x^{*}\right)^{T} \widetilde{Q} x^{*}={ }_{\mathscr{A}} \widetilde{c}^{T} \bar{x}+(1 / 2)(\bar{x})^{T} \widetilde{Q} \bar{x}$.

Proof. Since $\widetilde{Q}$ is fuzzy number positive semidefinite and $\left(x^{*}, u^{*}\right)$ solves DFNQP (37), we have that $Q$ is positive semidefinite and $\left(x^{*}, u^{*}\right)$ is an optimal solution to problem (38). From Theorem 8.2.6 in [27], some $\bar{x} \in R^{n}$ (not necessarily equal to $\left.x^{*}\right)$, satisfying $Q\left(\bar{x}-x^{*}\right)=0$, solves FNQP (11), and $-b^{T} u^{*}-(1 / 2)\left(x^{*}\right)^{T} Q x^{*}=c^{T} \bar{x}+(1 / 2)(\bar{x})^{T} Q \bar{x}$. From Theorem 7 above, $\bar{x}$ solves FNQP (36) and $-\widetilde{b}^{T} u^{*}-$ $(1 / 2)\left(x^{*}\right)^{T} \widetilde{Q} x^{*}={ }_{\mathscr{A}} \widetilde{c}^{T} \bar{x}+(1 / 2)(\bar{x})^{T} \widetilde{Q} \bar{x}$.

\section{Conclusion}

We used a linear ranking function to define the dual of fuzzy number quadratic programming primal problems. We provide optimality conditions for fuzzy number quadratic programming. Similar to general quadratic programming, we presented several duality results.

\section{Conflict of Interests}

The authors declare that there is no conflict of interests regarding the publication of this paper.

\section{Acknowledgments}

The authors thank the PhD Start-up Fund of Natural Science Foundation of Guangdong Province, China (no. S2013040012506) and Project Science Foundation of Guangdong University of Finance (no. 2012RCYJ005) for their support.

\section{References}

[1] L. L. Abdel-Malek and N. Areeratchakul, "A quadratic programming approach to the multi-product newsvendor problem with side constraints," European Journal of Operational Research, vol. 176, no. 3, pp. 1607-1619, 2007.

[2] E. Ammar and H. A. Khalifa, "Fuzzy portfolio optimization a quadratic programming approach," Chaos, Solitons and Fractals, vol. 18, no. 5, pp. 1045-1054, 2003.

[3] W.-G. Zhang and Z.-K. Nie, "On admissible efficient portfolio selection policy," Applied Mathematics and Computation, vol. 169, no. 1, pp. 608-623, 2005.

[4] T. Dwyer, Y. Koren, and K. Marriott, "Drawing directed graphs using quadratic programming," IEEE Transactions on Visualization and Computer Graphics, vol. 12, no. 4, pp. 536-548, 2006.

[5] J. A. M. Petersen and M. Bodson, "Constrained quadratic programming techniques for control allocation," IEEE Transactions on Control Systems Technology, vol. 14, no. 1, pp. 91-98, 2006.

[6] L. Pavlović and T. Divnić, "A quadratic programming approach to the Randić index," European Journal of Operational Research, vol. 176, no. 1, pp. 435-444, 2007. 
[7] H.-G. Schwarz, "Economic materials-product chain models: current status, further development and an illustrative example," Ecological Economics, vol. 58, no. 2, pp. 373-392, 2006.

[8] R. E. Bellman and L. A. Zadeh, "Decision-making in a fuzzy environment," Management Science, vol. 17, pp. B141-B164, 1970.

[9] Y.-J. Lai and C. L. Hwang, Fuzzy Mathematical Programming, vol. 394, Springer, Berlin, Germany, 1992.

[10] H. J. Zimmermann, "Description and optimization of Fuzzy system," International Journal of General Systems, vol. 2, no. 4, pp. 209-215, 1976.

[11] H.-J. Zimmermann, Fuzzy Set Theory and Its Applications, Kluwer-Nijhoff, Boston, Mass, USA, 1996.

[12] M. K. Luhandjula, "Fuzzy optimization: an appraisal," Fuzzy Sets and Systems, vol. 30, no. 3, pp. 257-282, 1989.

[13] S.-T. Liu, "Quadratic programming with fuzzy parameters: a membership function approach," Chaos, Solitons and Fractals, vol. 40, no. 1, pp. 237-245, 2009.

[14] L. A. Zadeh, "Fuzzy sets as a basis for a theory of possibility," Fuzzy Sets and Systems, vol. 1, no. 1, pp. 3-28, 1978.

[15] N. Mahdavi-Amiri and S. H. Nasseri, "Duality results and a dual simplex method for linear programming problems with trapezoidal fuzzy variables," Fuzzy Sets and Systems, vol. 158, no. 17, pp. 1961-1978, 2007.

[16] N. Mahdavi-Amiri and S. H. Nasseri, "Duality in fuzzy number linear programming by use of a certain linear ranking function," Applied Mathematics and Computation, vol. 180, no. 1, pp. 206216, 2006.

[17] J. F. Baldwin and N. C. F. Guild, "Comparison of fuzzy sets on the same decision space," Fuzzy Sets and Systems, vol. 2, no. 3, pp. 213-231, 1979.

[18] D. Dubois and H. Prade, "Ranking fuzzy numbers in the setting of possibility theory," Information Sciences, vol. 30, no. 3, pp. 183-224, 1983.

[19] P. Fortemps and M. Roubens, "Ranking and defuzzification methods based on area compensation," Fuzzy Sets and Systems, vol. 82, no. 3, pp. 319-330, 1996.

[20] K. Nakamura, "Preference relations on a set of fuzzy utilities as a basis for decision making," Fuzzy Sets and Systems, vol. 20, no. 2, pp. 147-162, 1986.

[21] J. Ramík and J. Rimanek, "Inequality relation between fuzzy numbers and its use in fuzzy optimization," Fuzzy Sets and Systems, vol. 16, no. 2, pp. 123-138, 1985.

[22] M. Roubens, "Comparison of at fuzzy numbers," in Proceedings of the North American Fuzzy Information Processing Society (NAFIPS '86), N. Badler and A. Kandel, Eds., pp. 462-476, 1986.

[23] C. Garcia-Aguado and J. L. Verdegay, "On the sensitivity of membership functions for fuzzy linear programming problems," Fuzzy Sets and Systems, vol. 56, no. 1, pp. 47-49, 1993.

[24] H. R. Maleki, M. Tata, and M. Mashinchi, "Linear programming with fuzzy variables," Fuzzy Sets and Systems, vol. 109, no. 1, pp. 21-33, 2000.

[25] M. Avriel, Nonlinear Programming Analysis and Methods, Prentice-Hall, Englewood Cliffs, NJ, USA, 1976.

[26] G. M. Lee, N. N. Tam, and N. D. Yen, Quadratic Programming and Affine Variational Inequalities: A Qualitative Study, Springer, New York, NY, USA, 2005.

[27] O. L. Mangasarian, Nonlinear Programming, vol. 10, Society for Industrial and Applied Mathematics, Philadelphia, Pa, USA, 1994. 


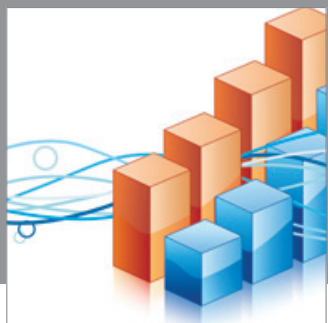

Advances in

Operations Research

mansans

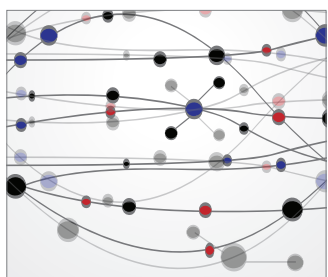

The Scientific World Journal
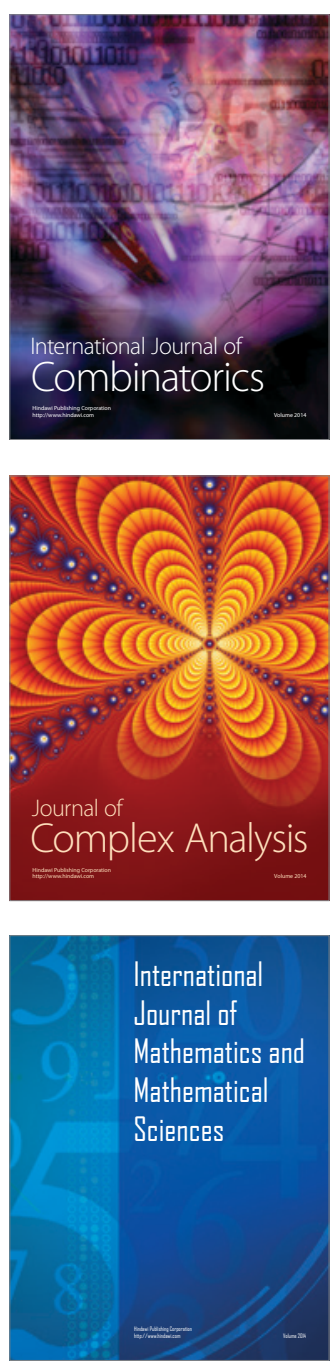
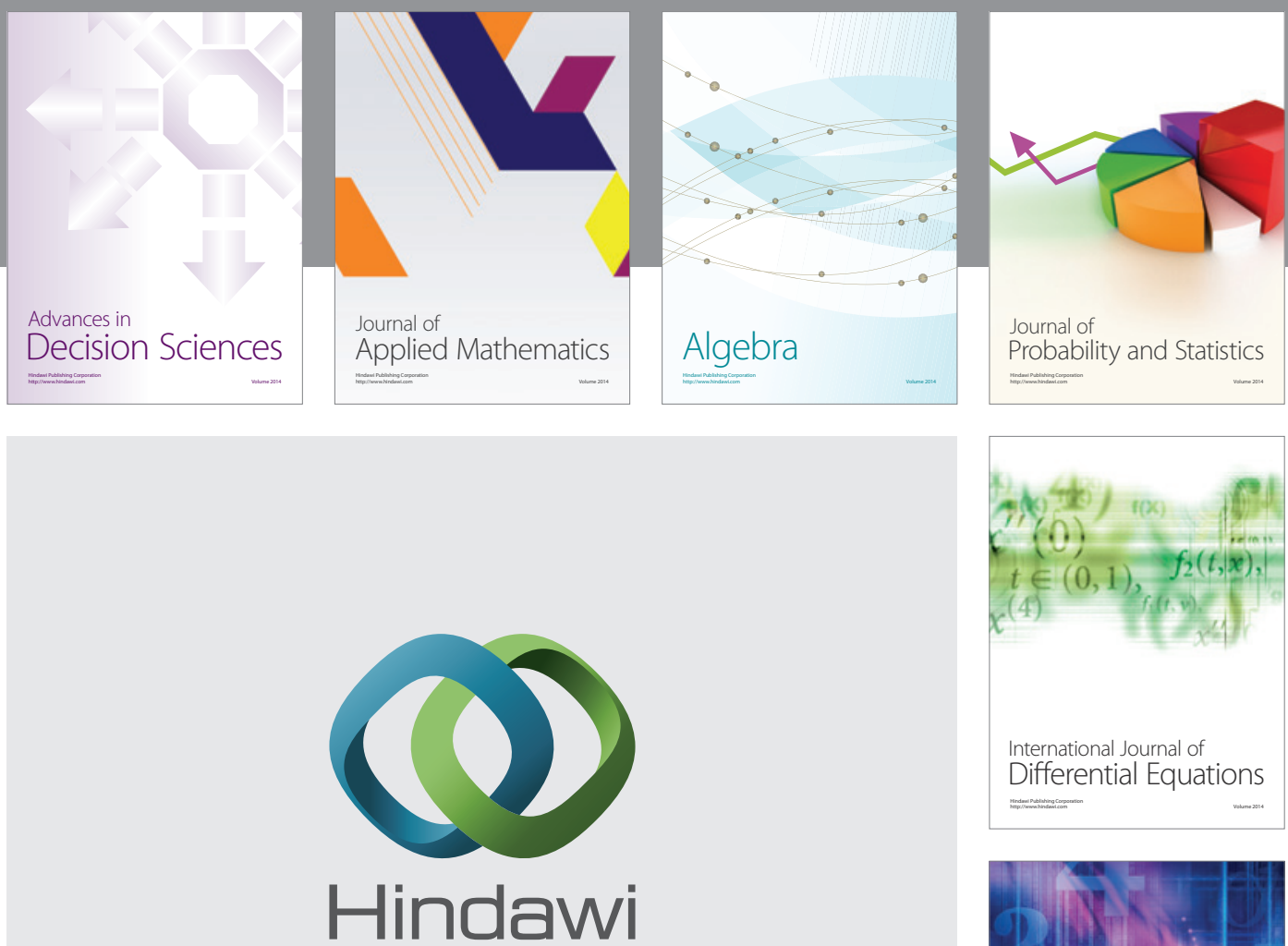

Submit your manuscripts at http://www.hindawi.com
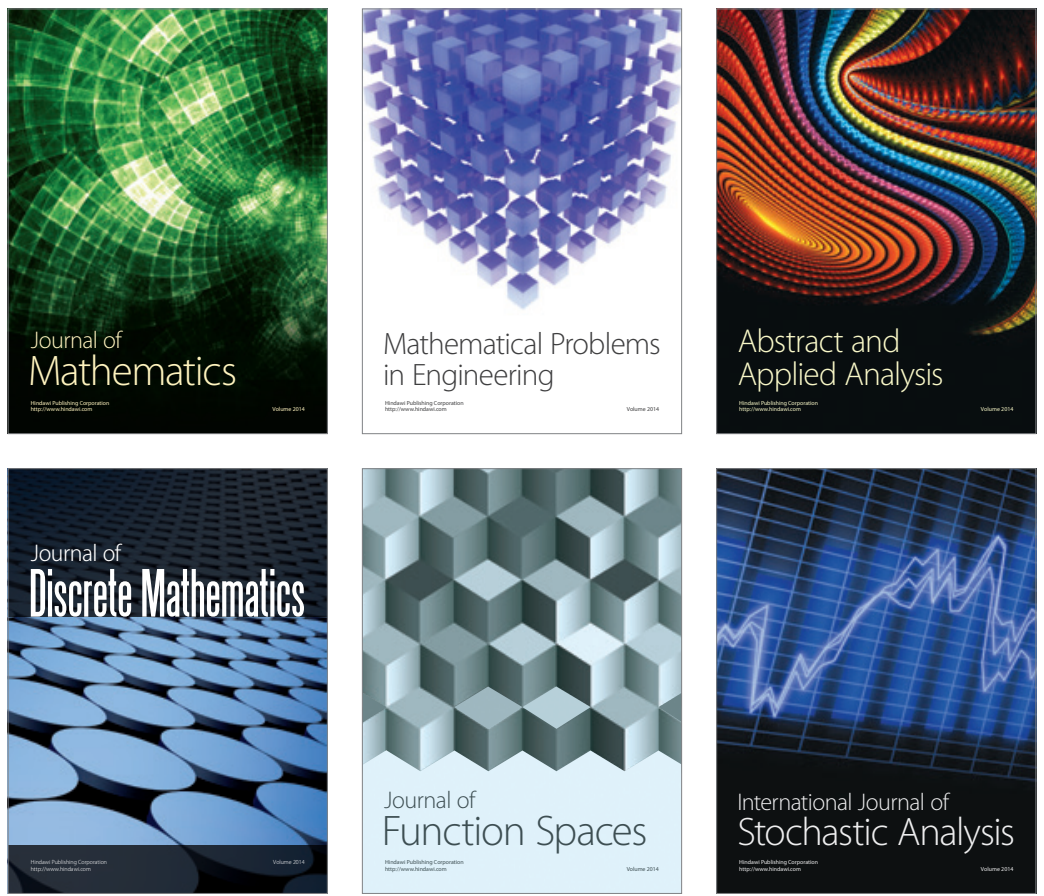

Journal of

Function Spaces

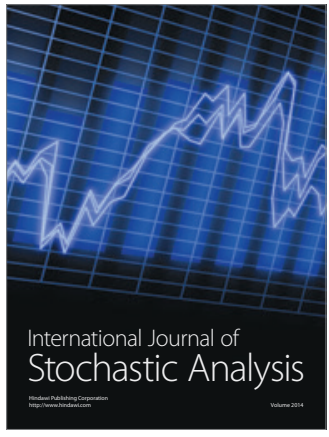

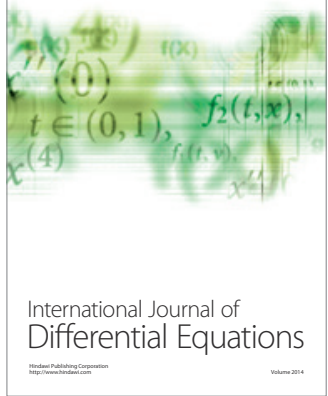
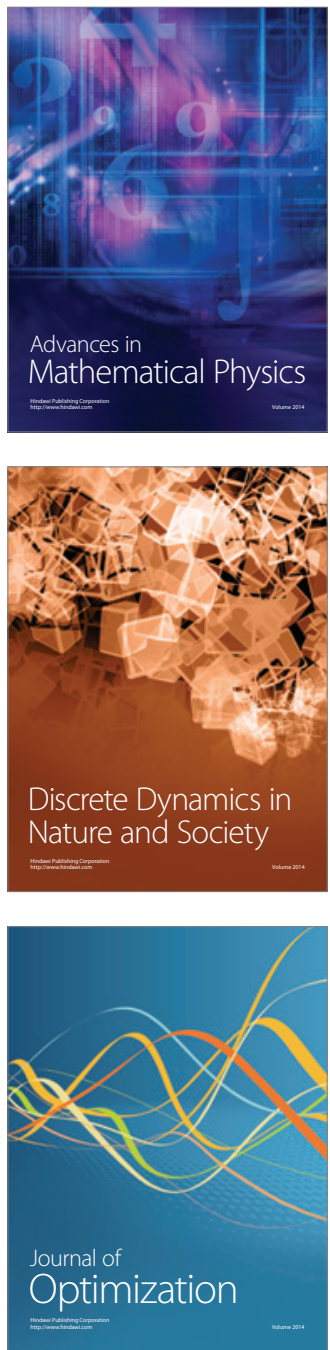